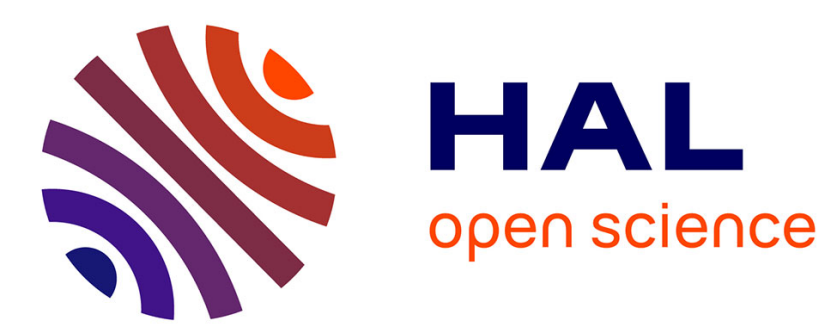

\title{
Extended criticality, phase spaces and enablement in biology
}

\author{
Giuseppe Longo, Maël Montévil
}

\section{To cite this version:}

Giuseppe Longo, Maël Montévil. Extended criticality, phase spaces and enablement in biology. Chaos, Solitons \& Fractals, 2013, Emergent Critical Brain Dynamics, 55, pp.64-79. 10.1016/j.chaos.2013.03.008 . hal-01192911v2

\section{HAL Id: hal-01192911 \\ https://hal.science/hal-01192911v2}

Submitted on 30 Jan 2017

HAL is a multi-disciplinary open access archive for the deposit and dissemination of scientific research documents, whether they are published or not. The documents may come from teaching and research institutions in France or abroad, or from public or private research centers.
L'archive ouverte pluridisciplinaire HAL, est destinée au dépôt et à la diffusion de documents scientifiques de niveau recherche, publiés ou non, émanant des établissements d'enseignement et de recherche français ou étrangers, des laboratoires publics ou privés. 


\title{
Extended Criticality, Phase Spaces and Enablement in Biology
}

\author{
Giuseppe Longo ${ }^{\mathrm{a}}$, Maël Montévil ${ }^{\mathrm{b}}$ \\ ${ }^{a}$ Centre Cavaillès, CNRS - École Normale Supérieure, Paris \\ ${ }^{b}$ Tufts University Medical School, Dept. of Anatomy and Cell Biology
}

\begin{abstract}
This paper analyzes, in terms of critical transitions, the phase spaces of biological dynamics. The phase space is the space where the scientific description and determination of a phenomenon is given. We argue that one major aspect of biological evolution is the continual change of the pertinent phase space and the unpredictability of these changes. This analysis will be based on the theoretical symmetries in biology and on their critical instability along evolution.

Our hypothesis deeply modifies the tools and concepts used in physical theorizing, when adapted to biology. In particular, we argue that causality has to be understood differently, and we discuss two notions to do so: differential causality and enablement. In this context constraints play a key role: on one side, they restrict possibilities, on the other, they enable biological systems to integrate changing constraints in their organization, by correlated variations, in un-prestatable ways. This corresponds to the formation of new phenotypes and organisms.
\end{abstract}

Keywords: Conservation properties, symmetries, biological causality, phase space, unpredictability, phylogenetic drift, enablement

\section{Introduction}

As extensively stressed by H. Weyl and B. van Fraassen, XXth century physics has been substituting to the concept of law that of symmetry. Thus, this concept may be "considered the principal means of access to the world we create in theories", Van Fraassen (1989).

In this text ${ }^{1}$, we will discuss the question of biological phase spaces in relation to critical transitions and symmetries. More precisely, we will argue, along the lines of Kauffman (2002; Bailly \& Longo (2008; Longo et al. (2012b), that in contrast to existing physical theories, where phase spaces are pre-given, in biology these spaces need to be analyzed as changing in unpredictable ways through evolution. This stems from the peculiar biological relevance of critical transitions and the related role of symmetry changes.

In order to understand the peculiarities of biological theorizing, we will first shortly recall the role, in physics, of "phase spaces". A phase space is the space of the pertinent observables and parameters in which the theoretical determination of the system takes place. As a result, to

\footnotetext{
${ }^{\text {Th }}$ Published as: Longo, G. \& Montevil, M. (2013). Extended criticality, phase spaces and enablement in biology. Chaos, Solitons $\mathcal{F}^{3}$ Fractals, 55 , 64-79. doi: 10.1016/j.chaos.2013.03.008.

Email addresses: longo@ens.fr (Giuseppe Longo), mael.montevil@gmail.com (Maël Montévil)

URL: http://www.di.ens.fr/users/longo (Giuseppe Longo), http://www montevil, theobio.org (Maël Montévil)

${ }^{1}$ This paper revises and develops early joint work with Stuart Kauffman Longo et al. (2012b).
}

one point of the phase space corresponds a complete determination of the intended object and properties that are relevant for the analysis.

Aristotle and Aristotelians, Galileo and Kepler closely analyzed trajectories of physical bodies, but without a mathematical theory of a "background space". In a sense, they had the same attitude as Greek geometers: Euclid's geometry is a geometry of figures with no space. It is fair to say that modern mathematical physics (Newton) begun by the "embedding" of Kepler and Galileo's Euclidean trajectories in Descartes' spaces. More precisely, the conjunction of these spaces with Galileo's inertia gave the early relativistic spaces and their invariant properties, as a frame for all possible trajectories - from falling bodies to revolving planets ${ }^{2}$. In modern terms, Galileo's symmetry group describes the transformations that preserve the equational form of physical laws, as invariants, when changing the reference system.

Along these lines, one of the major challenges for a (theoretical) physicist is to invent the pertinent space or, more precisely, to construct a mathematical space which contains all the required ingredients for describing the phenomena and to understand the determination of its trajectory, if any. So, Newton's analysis of trajectories was embedded in a Cartesian space, a "condition of possibility", Kant will explain, for physics to be done. By this, Newton unified (he did not reduce) Galileo's analysis of

\footnotetext{
${ }^{2}$ The Italian Renaissance painting invented the mathematical "background" space by the perspective, later turned into mathematics by Descartes and Desargues, see Longo (2011).
} 
falling bodies, including apples, to planetary orbits: Newton derived Kepler's ellipsis of a planet around the Sun from his equations. This is the astonishing birth of modern mathematical-physics as capable of predicting exactly the theoretical trajectory, once given the right space and the exact boundary conditions. But, since Poincaré, we know that if the planets around the Sun are two or more, prediction is impossible due to deterministic chaos. Even though their trajectories are fully determined by Newton-Laplace equations their non-linearity yields the absence almost everywhere of analytic solutions and forbids predictability, even along well determined trajectories at equilibrium.

As a matter of fact, Poincaré's analysis of chaotic dynamics was essentially based on his invention of the socalled Poincaré section (analyze planetary orbits only by their crossing a given plane) and by the use of momentum as a key observable. In his analysis of chaoticity, stable and unstable trajectories in the position-momentum phase space, nearly intersect infinitely often, in "infinitely tight meshes" and are also "folded upon themselves without ever intersecting themselves", (1892). Since then, in physics, the phase space is mostly given by all possible values of momentum and position, or energy and time. In Hamiltonian classical mechanics and in Quantum Physics, these observables and variables happen to be "conjugated", a mathematical expression of their pertinence and tight relation $^{3}$. These mathematical spaces are the spaces in which the trajectories are determined: even in Quantum Physics, when taking Hilbert's spaces as phase spaces for the wave function, Schrödinger's equation determines the dynamics of a probability density and the indeterministic aspect of quantum mechanics appears when quantum measurement projects the state vector (and gives a probability, as a real number value).

It is then possible to give a broader sense to the notion of phase space. For thermodynamics, say, Boyle, Carnot and Gay-Lussac decided to focus on pressure, volume and temperature, as the relevant observables: the phase space for the thermodynamic cycle (the interesting "trajectory") was chosen in view of its pertinence, totally disregarding the fact that gases are made out of particles. Boltzmann later unified the principles of thermodynamics to a particle's viewpoint and later to Newtonian trajectories by adding the ergodic hypothesis. Statistical mechanics thus, is not a reduction of thermodynamics to Newtonian trajectories, rather an "asymptotic" unification, at the infinite time limit of the thermodynamic integral, under the novel assumption of "molecular chaos" (ergodicity). In statistical mechanics, ensembles of random objects are considered as the pertinent objects, and observables are derived as aspects of their (parameterized) statistics.

It should be clear that, while the term phase space is often restricted to a position/momentum space, we use it here in the general sense of the suitable or intended space

\footnotetext{
${ }^{3}$ One is the position and the other takes into account the mass and the change of position.
}

of the mathematical and/or theoretical description of the system. In this sense the very abstract Hilbert space of complex probability densities is a phase space for the state function in Quantum Mechanics, very far form ordinary space-time.

Now, in biology, the situation is more difficult. Our claim here, along the lines of Kauffman (2002; Bailly \& Longo (2011; Longo et al. (2012b) is that, when considering the biologically pertinent observables, organisms and phenotypes, no conceptual nor mathematical construction of a pre-given phase space is possible for phylogenetic trajectories. This constitutes a major challenge in the study of biological phenomena. We will motivate it by different levels of analysis. Of course, our result is a "negative result", but negative results may open the way to new scientific thinking, in particular by the very tools proposed to obtain them, Longo (2012). Our tools are based on the role of symmetries and criticality, which will suggest some possible ways out.

\section{Phase Spaces and Symmetries}

We understand the historically robust "structure of determination of physics" (which includes unpredictability) by recalling that, since Noether and Weyl, physical laws may be described in terms of theoretical symmetries in the intended equations (of the "dynamics", in a general sense, see below). These symmetries in particular express the fundamental conservation laws of the physical observables (energy, momentum, charges ...), both in classical and quantum physics. And the conservation properties allow us to compute the trajectories of physical objects as geodetics, by extremizing the pertinent functionals (Hamilton principle applied to the Langrangian functionals). It is the case even in Quantum Mechanics, as they allow to derive the trajectory of the state function in a suitable mathematical space, by Schrödinger equation.

As we said, only with the invention of an (analytic) geometry of space (Descartes), could trajectories be placed in a mathematically pre-given space, which later became the absolute space of Newtonian laws. The proposal of the more general notion of "phase space" dates of the late XIX century. Then momentum was added to spatial position as an integral component of the analysis of a trajectory, or energy to time, in order to apply the corresponding conservation properties, thus the corresponding theoretical symmetries. In general, the phase spaces are the right spaces of description in the sense that they allow one to soundly and completely specify "trajectories": if one considers a smaller space, processes would not have a determined trajectory but would be able to behave arbitrarily with respect to the elements of the description (for example, ignoring the mass or the initial speed in classical mechanics). Adding more quantities would be redundant or superfluous (for example, considering the color or flavor, in the usual sense, in classical mechanics). 
In other words, in physics, the observables (and parameters), which form the phase space, derive from the (pertinent/interesting) invariants / symmetries in the trajectories and among trajectories. More exactly, they derive from the invariants and the invariant preserving transformations in the intended physical theory. So, Poincaré's momentum is preserved in the dynamics of an isolated system, similarly as Carnot's product $p V$ is preserved at constant temperature while $p$ and $V$ may vary. Again, one uses these invariants in order to construct the "background space" where the phenomena under analysis can be accommodated. That is, the conceptual construction of the phase space follows the choice of the relevant observables and invariants (symmetries) in the physico-mathematical analysis.

In summary, the historical and conceptual development of physics went as follows:

- analyze trajectories

- pull-out the key observables as (relative) invariants (as given by the symmetries)

- construct out of them the intended phase space.

Thus, physical (phase) spaces are not "already there", as absolutes underlying phenomena: they are our remarkable and very effective invention in order to make physical phenomena intelligible Weyl (1983; Bailly \& Longo (2011).

As H. Weyl puts it, the main lesson we learn from $\mathrm{XX}$ century physics is that the construction of scientific objectivity (and even of the pertinent objects of science) begins when one gives explicitly the reference system (or the phase space with its symmetries) and the metric (the measurement) on it. This is why the passage from the symmetries of Galileo group to Lorentz-Poincaré group frames Relativity Theory, as it characterizes the relevant physical invariants (the speed of light) and invariant preserving transformations (Poincaré group) in the phase space.

In summary, the modern work of the theoretical physicist begins by setting the phase space and the measure in it, on the grounds of the observables he/she considers to be essential for a complete description of the intended dynamics - in the broadest sense, like in Quantum Physics, where quanta do not go along trajectories in ordinary space-time, but the wave or state function does, in a Hilbert space.

As for the formal foundation, from Descartes' spaces up to the later more general phase spaces (Hilbert spaces or alike), all these spaces are finitistically (axiomatically) describable, because of their symmetries. That is, their regularities, as invariants and invariant preserving transformations in the intended spaces (thus their symmetries), allow a finite description, even if they are infinite. Consider, say, a tri- (or more) dimensional Cartesian space, since Newton our preferred space for physics. It is infinite, but the three straight lines are given by symmetries (they are axes of rotations) and their right angles as well ${ }^{4}$ (right angles, says Euclid, are defined from the most symmetric figure you obtain when crossing two straight lines). When adding the different groups of transformations (the symmetries) that allow to relativize the intended spaces, one obtains the various physical theories that beautifully organize the inert matter, up to today.

Hilbert and Fock's spaces require a more complex but conceptually similar definition, in terms of invariants and their associated transformations. These invariants (symmetries) allow to handle infinity formally, possibly in the terms of Category Theory. Note that symmetries, in mathematics, have the peculiar status of being both invariant (structural invariants, say) and invariant preserving transformations (as symmetry groups). Symmetries thus allow to describe infinite spaces and mathematical structures, even of infinite dimension, in a very synthetic way, by the finitely many words of a formal definition and of a few axioms.

We will argue for the intrinsic incompressibility of the phase space of intended observables in biology: no way to present it a priori, as a time invariant system, by finitely many pre-given words.

\subsection{More lessons from Quantum and Statistical Mechan- ics}

As we observed, quantum mechanics takes as state function a probability density in possibly infinite dimensional Hilbert or Fock spaces. More generally, in quantum mechanics, the density matrix allows to deal also with phase spaces which are known only in part. In such cases, physicists work with the part of the state space that is known and the density matrix takes into account that the system can end up in an unknown region of the state space, by a component called "leakage term". The point is that this term interferes with the rest of the dynamics in a determined way, which allows us to capture theoretically the situation in spite of the leakage term.

In Quantum Field Theory (QFT) it is even more challenging: particles and anti-particles may be created spontaneously. And so one uses infinite dimensional Hilbert's spaces and Fock spaces to accommodate them. Of course, quanta are all identical in their different classes: a new electron is an electron ... they all have the same observable properties and underlying symmetries. Also, the analysis by Feynman diagrams allows us to provide the participation in the quantum state of each possible spontaneous creation and annihilation of particles (and, basically, the more complex a diagram is, the smaller its weight). The underlying principle is that everything that can happen, for a quantum system, happens, but only a limited number of possibilities are quantitatively relevant.

In statistical mechanics one may work with a randomly varying number $n$ of particles. Thus, the dimension of

\footnotetext{
${ }^{4}$ More generally, modern Category Theory defines Cartesian products in terms of a symmetric commuting diagrams.
} 
the state space stricto sensu, which is usually $6 n$, is not pre-defined. This situation does not, however, lead to particular difficulties because the possibilities are known (the particles have a known nature, that is relevant observables and equational determination) and the probabilities of each phase space are given ${ }^{5}$. In other terms, even if the exact finite dimension of the space may be unknown, it has a known probability - we know the probability it will grow by 1, 2 or more dimensions, and, most importantly, they are formally symmetric. The possible extra particles have perfectly known properties and possible states: the pertinent observables and parameters are known, one just misses: how many? And this becomes a new parameter ... (see for example Sethna (2006), for an introduction).

In these cases as well, the analysis of trajectories or the choice of the object to study (recall the role given to momentum or the case of the thermodynamic cycle or the probability density for QM) lead to the construction of the pertinent phase space, which contains the proper observables and parameters for the trajectories of the intended object. Then, as mentioned above, the symmetries of the theories allowed synthetic, even axiomatic, definitions of these infinite spaces, even with infinite or fluctuating dimensions. In other words, the finite description of these spaces of possibly infinite dimension, from Descartes to Quantum spaces, is made possible by their regularities: they are given in terms of mathematical symmetries. And, since Newton and Kant, physicists consider the construction of the (phase) space as an "a priori" of the very intelligibility of any physical process.

\subsection{Criticality and Symmetries}

Certain physical situations are particularly interesting with respect to phase spaces and their symmetries. We refer by this to critical transitions, where there is a change of global behavior of a system, which may be largely described in terms of symmetries changes.

For example, spin lattices phase transitions are understood, from a purely macroscopic point of view, as a change of phase space: a parameter (the order parameter which is the global field in this example) shifts from being degenerate (uniformly null) to finite, non zero quantities. In other words, a new quantity becomes relevant. From a microscopic point of view, this quantity, however, is not exactly new: it corresponds to aspects used for the description of the microscopic elements of the system (a field orientation, for example). In the equational determination of the system as a composition of microscopic elements, there is no privileged directions for this observable. In the disordered phase (homogeneous, in terms of symmetries), the order parameter, that is the average of

\footnotetext{
${ }^{5}$ In general, $n$ changes either because of chemical reactions, and it is then their rate which is relevant, or because the system is open, in which case the flow of particles is similar to an energetic flow, that is the number of particles plays the same role than energy: they are both fluctuating quantities obeying conservation laws.
}

the field, is 0. However, in the ordered phase, the state of the system has a global field direction and its average departs from 0 .

The appearance of this observable at the macroscopic level is understood thanks to an already valid observable at the microscopic level, and by changing macroscopic symmetries. That is, at the critical point, the point of transition, we have a collapse of the symmetry of the macroscopic orientations of the field (the symmetry is verified when the field is null). This change corresponds to the formation of a coherence structure which allows microscopic fluctuations to extend to the whole system and in fine lead to a non null order parameter, the global field, after the transition. The system at the transition has a specific determination, associated to this coherence structure. Depending on the dimension of space, this physical process can require a specific mathematical approach, the renormalization method, which allows to analyze the characteristic multi-scale structure of coherence, dominated by fluctuations at all scales, proper to critical situations. In all cases, this situation is associated to a singularity in the determination of the system, which stem from the order parameter changing from a constant to a non-zero value.

On the basis of physical criticality, the concept of $e x$ tended critical transition has been first proposed to account for the specific coherence of biological systems, with their different levels of organization Bailly (1991), see also Longo et al. (2012c). The notion of different levels of organization is rather polysemic. It refers usually to the epistemic structuring of an organism by different forms of intelligibility, thus, a fortiori and if mathematically possible, by different levels of determination or mathematical description (molecular cascades, cells' activities and interactions, tissues' structures, organs, organisms ....). In the context of extended criticality, however, we propose to objectivize levels of organization and especially the change of level by the mathematical breaking of the determination of the first level, by singularities. This approach sheds an original light on the notion of level of organization, as the new level correspond to a coupling between scales and not simply to a higher scale Longo et al. (2012c).

The core hypothesis of extended criticality is that, when physical systems have a mainly point-wise criticality ${ }^{6}$, organisms have ubiquitous critical points (dense in a viability space, for example). Note that the interval of extended criticality may be given with respect to any pertinent parameter. Its main properties along this line are given in Bailly \& Longo (2008, 2011). The different levels of organization in this context are presented by fractal or fractallike structures and dynamics, as proposed by Werner and others, see West (2006; Werner (2010). More recent applications of this concept may be found in Lovecchio et al. (2012). Note also that criticality enables a multi-scale heterogeneity to take place, that the constraints of a normal

${ }^{6}$ This critical point can be an attractor: this is the paradigm of self-organized criticality. 
state prevent, which is of interest for biological symmetry changes Werner (2010; Machta et al. (2011).

A crucial aspect of extended criticality is given by the role of symmetries and symmetry changes in biological dynamics, developed in Longo \& Montévil (2011a). The density of critical points leads to omnipresent symmetry changes. Now, this has consequences for the very constitution of the scientific object. Physical objects are generic inasmuch different objects with the same equational determination will behave in the same way, and this way is determined by the specific trajectory provided precisely by the equations, a geodetic in the intended phase space. It is these specific trajectories, possibly after some transformations, which allow to state that objects behave the same, both in the theory and in experiments (i.e. they have invariant properties). The trajectory is thus obtained by using theoretical symmetries (conservation principles, see above) and in fine it allows to define physical objects as generic because they are symmetric (they behave the same way).

In contrast to this core perspective in physics, we propose that biological objects do not have such stable symmetries, and, thus, that their trajectories are not specific: there are no sufficiently stable symmetries and corresponding invariants, as for phenotypes, which would allow to determine the evolutionary dynamics of the object. On the contrary, the object follows a possible evolutionary trajectory, which may be considered generic. Conversely, the biological object is not generic but specific, Bailly \& Longo (2011). And it is so, since it is determined by a historical cascade of symmetry changes, Longo \& Montévil (2011a). In our approach, the inversion of generic vs. specific is a core conceptual duality of biological theorizing vs. physical one. It deeply modifies the status of the object.

The starting assumption in this approach to evolutionary trajectories is based on Darwin's first principle (and default state for biology, Sonnenschein \& Soto (1999): Descent with modification. Darwin's other principle, selection, would make little sense without the first.

Notice that Darwin's first principle is a sort of nonconservation principle as for phenotypes (see 6): any reproduction yields (some) changes. It is crucial for us that this applies at each individual cellular mitosis. As a matter of fact, each mitosis may be seen as a critical transition. In a multicellular organism, in particular, it is a bifurcation that yields the reconstruction of a whole coherence structure: the tissue matrix, the collagen's tensegrity structure, the cells' dialogue in general. And this besides the symmetry breakings due to proteome and DNA variations, which we will further discuss. In short, in view of the "density" of mitoses in the life interval of an organism, we may already consider this phenomenon at the core of its analysis in terms of extended criticality.

In this context, the mathematical un-predefinability of biological phase space we discuss below will follow by comparing the physico-mathematical constructions to the needs of biology, where theoretical symmetries are not pre- served. Let us recall that we work in a Darwinian frame and consider organisms and phenotypes as the pertinent observables.

\section{Non-ergodicity and quantum/classical random- ness in biology.}

We will discuss here the issue of "ergodicity" as well as the combination of quantum and classical random phenomena in biology. By ergodicity, we broadly refer to Boltzmann's assumption in the 1870's that, in the course of time, the trajectory of a closed system passes arbitrarily close to every point of a constant-energy surface in phase space. This assumption allows to understand a system without taking into account the details of its dynamic.

From the molecular viewpoint, the question is the following: are (complex) phenotypes the result of a random exploration of all possible molecular combinations and aggregations, along a path that would (eventually) explore all molecular possibilities?

An easy combinatorial argument shows that at levels of complexity above the atom, for example for molecules, the universe is grossly non-ergodic, that is it does not explore all possible paths or configurations. Following an example in Kauffman (2002), the universe will not make all possible proteins length 200 amino acids in 10 to the 39th times its lifetime, even were all 10 to the 80th particles making such proteins on the Planck time scale. So, their "composition" in a new organ, function or organism (thus, in a phenotype) cannot be the result of the ergodicity of physical dynamics ${ }^{7}$.

The point is that the lack of ergodicity presents an immediate difficulty for the (naive) reductionist approach to the construction of a phase space for biological dynamics, as given in purely molecular terms. In order to understand this, let's consider the role of ergodicity in statistical mechanics. A basic assumption of statistical mechanics is a symmetry between states with the same energetic level, which allows to analyze their probabilities (on the relevant time scales). This assumption is grounded on a hypothesis of ergodicity as for the dynamics of the particles: at the limit of infinite time, they "go everywhere" in the intended phase space, and they do so homogeneously (with a regular frequency). In this case, the situation is described on the basis of energetic considerations (energy conservation properties, typically), without having to take into account the Newtonian trajectory or the history of the system.

In biology, non-ergodicity in the molecular phase space allows to argue that the dynamic cannot be described without historical considerations, even when taking only

\footnotetext{
${ }^{7}$ Notice here that this argument only states that ergodicity in the molecular phase space does not help to understand the biological dynamics of phenotypes. The argument does not preclude the trajectories from being ergodic in infinite time. We can then say that ergodicity is biologically irrelevant and can take this irrelevance as a principle.
} 
into account molecular aspects of biological systems. $A$ fortiori, this holds when considering morphological and other higher scale biological aspects (the phenotypes in the broadest sense). In other terms, non-ergodicity in biology means that the relevant symmetries depend on a history even in a tentative phase space for molecules, which is in contrast with (equilibrium) statistical mechanics.

To sum the situation up, non-ergodicity prevent us to symmetrize the possible. With respect to a Darwinian phase space, most complex things will never exist and don't play a role, Kauffman (2002). The history of the system enters into play and canalizes evolution.

Note that some cases of non-ergodicity are well studied in physics. Symmetry breaking phase transitions is a simple example: a crystal does not explore all its possible configurations because it has some privileged directions and it "sticks" to them. The situation is similar for the magnetization of a magnet (see Strocchi (2005) for a mathematical analysis). A more complex case is given by glasses. Depending on the models, the actual non-ergodicity is valid either for infinite time or is only transitory, yet relevant at the human time scales. Crucially, non-ergodicity corresponds to a variety of possible states, which depend on the paths in the energetic landscape that are taken (or not taken) during the cooling. This can be analyzed as an entropic distance to thermodynamic equilibrium and corresponds to a wide variety of "choices". However, the various states are very similar and their differences are relatively well described by the introduction of a time dependence for the usual thermodynamic quantities. This corresponds to the so-called "aging dynamics" Jensen \& Sibani (2007). The example of glassy dynamics show that the absence of a relevant ergodicity is not sufficient in order to obtain phase space changes in the sense we will describe, because in this example the various states can be understood in an a priori well-defined phase space and are not qualitatively different.

Note, finally, that an ergodic trajectory is a "random", yet complete, exploration of the phase space. However, ergodicity does not coincide with randomness, per se: a step-wise random trajectory (i.e. each step at finite time is random), does not need to be ergodic, since ergodicity, in mathematical physics, is an asymptotic notion.

Now, biological dynamics are a complex blend of contingency (randomness), history and constraints. Our thesis here is that biological (constrained) randomness is essential to variability, thus to diversity, thus to life.

The most familiar example is provided by meiosis, as gametes randomly inherit chromosomes pairs from the parents. Moreover, chromosomes of a given pair may exchange homologous portions and, so far, this is analyzed in purely probabilistic terms. It is a well established fact that DNA recombinations are a major contribution to diversity. However, all aspects of meiosis depend on a common history of the mixing DNA's and viable diversity is restricted by this history.

A finer analysis can be carried on, in terms of ran- domness. In a cell, classical and quantum randomness both play a role and "superpose". Recall first that, in physics, classical and quantum randomness differ: different probability theories (thus measures of randomness) may be associated to classical events vs. (entangled) quantum events. Bell inequalities distinguishes them (see Aspect et al. (1982)).

Some examples of biologically relevant quantum phenomena are electron tunneling in cellular respiration Gray \& Winkler (2003), electron transport along DNA Winkler et al. (2005), quantum coherence in photosynthesis Engel et al. (2007; Collini et al. (2010). Moreover, it has been shown that double proton transfer affects spontaneous mutation in RNA duplexes Ceron-Carrasco et al. (2009). The enthalpic chaotic oscillations of macro-molecules instead have a classical nature, in physical terms, and are essential to the interaction of and with DNA and RNA. Quantum randomness in a mutation is typically amplified by classical dynamics (including classical randomness), in the interaction between DNA, RNA and the proteome (see Buiatti \& Longo (2013) for a discussion). This kind of amplification is necessary in order to understand that changes at the nanometer scale impact the phenotype of the cell or of the organism. Moreover, it may be sound to consider the cell-to-cell interactions and, more generally, ecosystem's interactions as classical, at least as for their physical aspects, yet affecting the biological observables, jointly with quantum phenomena.

Poincaré discovered the destabilizing effects of planetary mutual interactions, in particular due to gravitational resonance (planets attract each other, which cumulates when aligned with the Sun); by this, in spite of the deterministic nature of their dynamics, they go along unstable trajectories and show random behavior, in astronomical times (see Laskar J. (1994)). In Buiatti \& Longo (2013), by analogy, the notion of "bio-resonance" is proposed. Different levels of organization, in an organism, affect each other, in a stabilizing (regulating and integrating), but also in a destabilizing way.

A minor change in the hormonal cascade may seriously damage a tissue's coherence and, years later, cause or enable cancer. A quantum event at the molecular level (a mutation) may be amplified by cell to cell interaction and affect the organism, whose changes may downwards affect tissues, cells, metabolism. Note that Poincaré's resonance and randomness are given at a unique and homogeneous level of organization (actually, of mathematical determination). Bio-resonance instead concerns different epistemic levels of organization, thus, a fortiori and if mathematically possible, different levels of determination or mathematical description (molecular cascades, cells, tissues, organs, organisms ...).

In evolution, when a (random) quantum event at the molecular level (DNA or RNA-DNA or RNA-protein or protein-protein) happens to have consequences at the level of the phenotype, the somatic effects may persist if they are inherited and compatible both with the ever changing 
ecosystem and the "coherence structure" of the organism, that is, when they yield viable Darwin's correlated variations. In particular, this may allow the formation of a new function, organ or tool or different use of an existing tool, thus to the formation of a new properly relevant biological observable (a new phenotype or organism). This new observable has at least the same level of unpredictability as the quantum event, but it does not belong to the quantum phase space: it is typically subject to Darwinian selection at the level of the organisms in a population, thus it interacts with the ecosystem as such. Recall that this is the pertinent level of observability, the level of phenotypes, where biological randomness and unpredictability is now to be analyzed.

We stress again that the effects of the classical / quantum blend may show up at different levels of observability and may induce retroactions. First, as we said, a mutation or a random difference or expression in the genome, may contribute to the formation of a new phenotype ${ }^{8}$. Second, this phenotype may retroact downwards, to the molecular (or quantum) level. A molecular activity may be excluded, as appearing in cells (organs / organisms) which turn out to be unfit - selection acts at the level of organisms, and may then exclude molecular activities associated to the unfit organism. Moreover, methylation and de-methylation downwards modify the expression of "genes". These upwards and downwards activities contribute to the integration and regulation of and by the whole and the parts. They both contribute to and constrain the biological dynamics and, thus, they do not allow to split the different epistemic levels of organization into independent phase spaces.

We recall that our choice of the biologically pertinent observables is based on the widely accepted fact that nothing makes sense in biology, if not analyzed in terms of evolution. We summarized the observables as the "phenotype", that is, as the various (epistemic) components of an organism (organs, tissues, functions, internal and ecosystemic interactions ....).

Thus, evolution is both the result of random events at all levels of organization of life and of constraints that canalize it, in particular by excluding, by selection, incompatible paths - where selection is due both to the interaction with the ecosystem and the maintenance of a possibly renewed internal coherent structure of the organism, constructed through its history. So, ergodic explorations are restricted or prevented both by selection and by the history of the organism (and of the ecosystem). For example, the presence and the structure of a membrane, or a nucleus, in a cell canalizes also the whole cellular activities along a restricted form of possible dynamics ${ }^{9}$.

\footnotetext{
${ }^{8}$ In some bacteria, the lac-operon control system may be inherited at the level of proteome, see Robert et al. (2010).

${ }^{9}$ See Machta et al. (2011) for an analysis of the molecular spatial heterogeneity in the membrane as enabled by the coupling of phase transition fluctuations and the cytoskeleton.
}

In conclusion, the "canalizing" role of history and selection, which excludes what is incompatible with the ecosystem and/or with the internal coherence of the organism, coexists with the various forms of randomness we mentioned. We find it critical that neither quantum mechanics alone, nor classical physics alone, account for evolution. Both seem to work together. Mutations and other molecular phenomena may depend on random, acausal, indeterminate quantum events. Thus they may interfere or happen simultaneously to or be amplified by classical dynamics, as well as by phenotype - phenotype interaction. In this amplification, evolution is also not completely random, as seen in the similarity of the octopus and vertebrates' camera eye, independently evolved (see below). Thus evolution is both strongly canalized (or far from ergodic) and yet indeterminate, random and acausal. Our key point is then that random events, in biology, do not "just" modify the (numerical) values of an observable in a pre-given phase space, like in physics. They modify the very phase space, or space of pertinent biological (evolutionary) observables, the phenotypes.

\section{Symmetries breakings and randomness}

We propose that in all existing physical theories each random event is associated to a symmetry change. This is a preliminary, still conjectural remark, yet it may turn out to be important when stressing the role of randomness in biology.

A random event is an event where the knowledge about a system at a given time does not entail its future description. In physics though, the description before the event determines the complete list of possible outcomes: these are numerical values of pre-given observables - modulo some finer considerations as the ones we made as for QM and statistical physics, on the dimensions of the phase space, typically. Moreover, in most physical cases, the theory provides a metric (probabilities or other measures) which determines the observed statistics (random or unpredictable, but not so much: we know a probability distribution). Kolmogorov's axiomatic for probabilities work this way and provide probabilities for the outcomes. The different physical cases can be understood and compared in terms of symmetry breaking.

- In Quantum Mechanics, the unitarity of the quantum evolution is broken at measurement, which amounts to say that the quantum state space assumes privileged directions (a symmetry breaking).

- In classical probabilities, the intended phase space contains the set of all possibilities. Elements of this set are symmetric inasmuch they are possible, moreover the associated probabilities are usually given by an assumption of symmetry, for example the sides of a dice (or the regions of the phase space with the same energy). These symmetries are broken by drawing, which singles out a result. 
- Algorithmic concurrency theory states the possibilities but do not provide, a priori, probabilities for them. These may be added if the physical event forcing a choice is known (but computer scientists usually "do not care" — this is the terminology they use, see Longo et al. (2010)).

In physical theories, we thus associated a random event to a symmetry breaking. In each case, we have several possible outcomes that have therefore a symmetrical role, possibly measured by different probabilities. After the random event, however, one of the "formerly possible" events is singled out as the actual result. Therefore, each random event that fits this description is based on a symmetry breaking, which can take different yet precise mathematical forms, depending in particular on the probability theory involved (or lack thereof).

Let's now more closely review, in a schematic way, how the random events are associated to symmetry breakings:

Quantum Mechanics: the projection of the state vector (measurement); non-commutativity of measurement; tunneling effects; creation of a particle ....

Classical dynamics: bifurcations, for example, correspond typically to symmetric solutions for periodic orbits. Note that in classical mechanics, "the knowledge of the system at a given time" involve the measurement (inasmuch it limits the access to the state) and not only the state itself.

Critical transitions: the point-wise symmetry change lead to a "choice" of specific directions (the orientation of a magnet, the spatial orientation of a crystal, etc.). The specific directions taken are associated to fluctuations. Also, the multi-scale configuration at the critical point is random, and fluctuating.

Thermodynamics: the arrow of time (entropy production). This case is peculiar as the randomness and the symmetry breaking are not associated to an event but to the microscopic description. The time reversal symmetry is broken at the thermodynamic limit. Also, the evolution is towards a symmetrization of the system, since it tends towards the macroscopic state to which correspond the greatest number of microscopic states (they are symmetric from a macroscopic viewpoint), that is the greatest entropy, compatible with other constraints.

Algorithmic concurrency: The choice of one of the possible computational paths (backtracking is impossible).

If this list is exhaustive, we may say that random events, in physics, are correlated to symmetry breakings. These symmetry changes and the associated random events happen within the phase space given by the intended physical theory.
The challenge we are facing, in biology, is that randomness, we claim, manifests itself at the very level of the observables: randomness breaks theoretical symmetries and modifies the very phase space of evolution. Critical transitions are the closest physical phenomenon to the needs of the theoretical investigation in biology. Yet, the change of physical observables is given within a uniform theoretical frame. The new macroscopic observable, after the transition, is already used for the description of the elements of the system. At the critical point, the system may be described by a cascade of parametrized models, whose parameter, the scale, lead the sequence to converge to a new, but predictable coherent structure, with a specific scale symmetry. Moreover, the process may be sometimes reversed, always iterated; when iterated, fluctuations close to transition may at most give quantitative differences in the symmetries obtained at the transition.

The perspective that we advocate in biology differs then from the physical cases, and takes care of dynamics where the possible outcomes, as defining properties of biological observables, cannot be entailed from the knowledge of the system. There is no such situation to our knowledge in physics: the so frequently claimed "emergence" of water properties from quantum properties, Longo et al. (2015), or of relativistic field from the quantum one, confirms our claim. In these cases, physicists, so far, change symmetries so radically that they need to change theory. Hydrodynamics, for example, deals with observable properties of water such as fluidity and incompressibility in continua, which are symmetries far away from those of QM. And the challenge is to invent a third theory, framing the existing incompatible ones, a "unification" as people say in field theory ${ }^{10}$. Note that a common way to go from a theory to another is to use asymptotic reasonning, that is to say, to consider that some quantity goes to infinity, which alters the symmetry of a situation Bailly \& Longo (2011; Batterman (2007).

\section{Randomness and phase spaces in biology}

As hinted above, we understand randomness in full generality as unpredictability with respect to the intended theory. This is of course a relativized notion, as the practice of physics shows, for example in the quantum vs. classical randomness debate. In either case, randomness is "measurable" and its measure is given by probability theory. As a matter of fact, in pre-given spaces of possibilities (the pertinent phase spaces), modern probability theory may be largely seen as a specific case of Lebesgue Measure Theory.

\footnotetext{
${ }^{10}$ Einstein tried very hard to reduce the Quantum Field to the relativistic one, or to have the first theoretically "emerge" from the second, by claiming its incompleteness, if not completed by relativistic (hidden) variables. And he deduced and discovered quantum entanglement, by the mathematical investigation in Einstein et al. (1935); a positive consequence of a negative result.
} 
More precisely, the measure (the probabilities) is given in terms of (relative) probabilities defined by symmetries with respect to the observable in a prestated phase space, as for the 6 symmetric faces of a fair dice. A more sophisticated example is the microcanonical ensemble of statistical mechanics, where the microstates with the same energy have the same probability (are symmetric or interchangeable), on the grounds of the ergodic hypothesis. In either case, the random event results in a symmetry breaking: one out of the six possible (symmetric) outcomes for a dice; the random exploration of a specific microstate in statistical mechanics (see section 4 above).

Recall that, by "theoretical symmetries", in biology, we refer both to the phenomenal symmetries in the phenotype and to the "coherence structure" of an organism, a niche, an ecosystem, in the broadest sense. In some cases, these symmetries may be possibly expressed by balance equations, at equilibrium or far from equilibrium, like in physics, or just by the informal description of its working unity as balanced processes of functions, organs and global autopoietic dynamics Varela et al. (1974; Mossio \& Moreno (2010). Under all circumstances, a permanent exploration and change is at the core of biology, or, as Heraclitus and Stuart Kauffman like to say: "Life bubbles forth". Yet, it does so while struggling to preserve its relative stability and coherence.

We need to understand this rich and fascinating interplay of stabilities and instabilities. Extended critical transitions in intervals of viability, the associated symmetry changes and bio-resonance are at the core of them: they yield coherence structures and change them continually, through epistemic levels of organization. Bio-resonance integrates and regulates the different levels within an organisms, while amplifying random effects due to transitions at one given level. At other levels of organization, these random events may yield radical changes of symmetries, coherent structures and, eventually, observable phenotypes.

In biology, randomness enhances variability and diversity. It is thus at the core of evolution: it permanently gives diverging evolutionary paths, as theoretical bifurcations in the formation of phenotypes. We also stressed that variability and diversity are key components of the structural stability of organisms, species and ecosystems, alone and together. Differentiation and variability within an organism, a species and an ecosystem contribute to their diversity and robustness, which, in biology, intrinsically includes adaptiveness. Thus, robustness depends also on randomness and this by low numbers: the diversity in a population, or in an organ, which is essential to their robustness, may be given by few individuals (organisms, cells). This is in contrast to physics, where robustness by statistical effects inside a system is based on huge numbers of elementary components, like in thermodynamics, in statistical physics and in quantum field theory Lesne (2008). Actually, even at the molecular level, the vast majority of cell proteins are present in very low copy numbers, so the variability due to proteome (random) differences after a mitosis, yields new structural stabilities (the new cells) based on low but differing numbers.

Moreover, there exists a theoretical trend of increasing relevance that considers gene expression as a stochastic phenomenon. The theory of stochastic gene expression, usually described within a classical frame, is perfectly compatible, or it actually enhances our stress on randomness and variability, from cell differentiation to evolution ${ }^{11}$. In those approaches, gene expression must be given in probabilities and these probabilities may depend on the context (e. g. even the pressure on an embryo, see Brouzés \& Farge (2004)). This enhances variability even in presence of a stable DNA.

Besides the increasingly evident stochasticity of gene expression, contextual differences may also force very different uses of the same (physical) structure. For example, the crystalline in a vertebrate eye and the kidney and their functions use the same protein Michl et al. (2006), with different uses in these different context. Thus, if we consider the proper biological observable (crystalline, kidney), each phenotypic consequence or set of consequences of a chemical (enzymatic) activity has an a priori indefinite set of potential biological uses: when, in evolution, that protein was first formed, there was no need for life to build an eye with a crystalline. There are plenty of other way to see, and animals do not need to see. Similarly, a membrane bound small protein, by Darwinian pre-adaptation or Gould's exaptation, may become part of the flagellar motor of a bacterium, while originally it had various, unrelated, functions Liu \& Ochman (2007). Or, consider the bones of the double jaw of some vertebrates that evolved into the bones of the middle ears of mammals (one of Gould's preferred examples of exaptation), see Allin (1975). A new function, hearing, emerged as the "bricolage" (tinkering) of old structures. There was no mathematical necessity for the phenotype nor for the function, "listening", in the physical world. Indeed, most complex things do not exist in the Universe, as we said.

Evolution may also give divergent answers to the same or to similar physical constraints. That is, the same function, moving, for example, or breathing, may be biologically implemented in very different ways. Trachea in insects versus vertebrates' lungs (combined with the vascular system), are due both to different contexts (different biological internal and external constraints) and to random symmetry changes in evolutionary paths. Thus, very different biological answers to the "same" physical context make phenotypes incomparable, in terms of physical optima: production of energy or even exchanging oxygen may be dealt with in very different ways, by organisms in the "same" ecosystem.

Conversely, major phenomena of convergent evolution

\footnotetext{
${ }^{11} \mathrm{~A}$ pioneering paper on this perspective is Kupiec (1983): recent surveys may be found in Paldi (2003; Arjun \& van Oudenaarden (2008; Heams (2014).
} 
shape similarly organs and organisms. Borrowing the examples in Longo et al. (2012b), the convergent evolutions of the octopus and vertebrate eye follow, on one side, random, possibly quantum based acausal and indeterminate mutations, which contributed to very different phylogenetic paths. On the other, it is also "not-so-random" as both eyes converge to analogous physiological structures, probably due to physical and biological similar constraints - acting as co-constituted borders or as selection. The convergent evolution of marsupial and mammalian forms, like the Tasmanian wolf (a marsupial) and mammalian wolf are other examples of convergent, not-so-random components of evolution, in the limited sense above.

In conclusion, randomness, in physics, is "constrained" or mathematically handled by probabilities, in general with little or no relevance of history, and by possibly decorrelating events from contexts. In biology, histories and contexts (sometime strongly) canalize and constraint random evolutions.

That is, randomness may be theoreticaly constrained, in physics, by probability values in a pre-given list of possible future events; in biology, it is constrained by the past history and the context of an event.

\subsection{Non-optimality}

Given the lack of ordered or orderable phase spaces, where numbers associated to observables would allow comparisons, it is hard to detect optimality in biology, except for some local organ construction. In terms of physical or also biological observables, the front legs of an elephant are not better nor worst than those of a Kangaroo: front podia of tetrapodes diverged (broke symmetries differently) in different biological niches and internal milieu. And none of the issuing paths is "better" than the other, nor followed physical optimality criteria, even less biological ones: each is just a possible variation on an original common theme, just compatible with the internal coherence and the coconstituted ecosystem that enabled them.

In general, thus, there is no way to define a real valued (Lagrangian) functional to be extremized as for phenotypes, as this would require an ordered space (a real valued functional), where "this phenotype" could be said to be "better" than "that phenotype". The exclusion of the incompatible, in given evolutionary context, in no ways produces the "fittest" or "best", in any physicomathematical rigorous sense. Even Lamarckian effects, if they apply, may contribute to fitness, not to "fitter", even less "fittest". Only "a posteriori" can one say that "this is better than that" (and never "best" in an unspecified partial upper semi-lattice): the a posteriori trivial evidence of survival and successful reproduction is not an a priori judgment, but an historical one. Dinosaurs dominated the Earth for more than 100 millions years, leaving little ecological space to mammals. A meteor changed evolution by excluding dinosaurs from fitness: only a posteriori, after the specific consequences of that random event, mammals may seem better - but do not say this to the mammals then living in Yucatan. The blind cavefish, an "hopeful monster" in the sense of Goldschmidt, a posteriori seems better than the ascendent with the eyes, once it adapted to dark caverns by increasing peripheral sensitivity to water vibrations. This a priori incomparability corresponds to the absence of a pre-given partial order among phenotypes, thus of optimizing paths, simply because their space is not pre-given. At most, sometimes, one can make a pair-wise a posteriori comparisons (which is often associated to experimental situation, with controled, simple conditions). This incomparability is also due to the relative independence of niches, which are co-constituted by organisms.

More generally, conservation or optimality properties of physical observables (the various forms of physical energy, for example) cannot help to determine the evolutionary trajectory of an organism. No principle of "least free energy" (or "least time consumption of free energy", if it applies) can help to predict or understand completely the evolution of a proper and specific biological observable, nor of an organism as object of selection. A given physical ecosystem may yield very different organisms and phenotypes. A Darwin says, reproduction always implies variation, even without being prompted by the environment.

Physical forces may help to determine the dynamics only locally, for example the form of some organs, where exchange of matter or energy dominates (lungs, vascular system, phyllotaxis ...). Their forms partly follow optimality principles (dynamical branching, sprouting or fractal structures or alike, see Jean (1994; Fleury (2000; Bailly et al. (1988)). In these cases, physical forces (the pushing of the embryonal heart, respiration ..., tissue matrix frictions ...) are fundamental dynamical constraints to biology's default state: proliferation with variation and motility. Then selection applies at the level of phenotypes and organisms. Thus the result is incompletely understood by looking only at the physical dynamical constraints, since variability and diversity (the irregularity of lungs, of plants organs in phyllotaxis ...) contribute to robustness. They are not "noise" as in crystals' formation.

\section{A non-conservation principle}

The phylogenetic drift underlying evolution must be understood in terms of a "non-conservation principle" of biological observables. Darwin proposed it as a principle, to which we extensively referred: descent with modification, on which selection acts. This is the exact opposite of the symmetries and conservation properties that govern physics and the related equational and causal approaches. There is of course structural stability, in biology, which implies similar, but never identical iteration of a morphogenetic process. Yet, evolution requires also and intrinsically this non-conservation principle for phenotypes in order to be made intelligible. In particular, one needs to 
integrate randomness, variability and diversity in the theory in order to understand phylogenetic and ontogenetic adaptability and the permanent exploration and construction of new niches.

In a sense, we need, in biology, a similar enrichment of the perspective as the one quantum physicists dared to propose in the '20th: intrinsic indetermination was introduced in the theory by formalizing the non-commutativity of measurement (Heisenberg non-commutative algebra of matrices) and by Schrödinger equation (the deterministic dynamics of a probability law). We propose here an analysis of indetermination at the level of the very formation of the phase space, or spaces of evolutionary possibilities, by integrating Darwin's principle of reproduction with modification and, thus, of variability, in the intended structure of determination.

As a further consequence, the concept of randomness in biology we are constructing mathematically differs from physical forms of randomness, since we cannot apply a probability measure to it, in absence of a pre-given space of possible phenotypes in evolution (nor, we should say, in ontogenesis, where monsters appear, sometimes hopeful from the point of view of evolution). The lack of probability measures may resemble the "do not care" principle in algorithmic concurrency, over computer networks, mentioned above (and networks are fundamental structures for biology as well). However, the possible computational paths are pre-given and, moreover, processes are described on discrete data types, which are totally inadequate to describe the many continuous dynamics present in biology. Indeed, the sequential computers, in the nodes, are Laplacian Discrete State machine, as Turing first observed Longo et al. (2012a), far away from organisms.

In summary, in biology, the superposition of quantum and classical physics, bio-resonance, the coexistence of indeterminate acausal quantum molecular events, with somatic effects, and of non-random historical and contextual convergences do not allow to invent, as physicists do, a mathematically stable, pre-given phase space, as a "background" space for all possible evolutionary dynamics.

Random events break symmetries of biological trajectories in a constitutive way. A new phenotype, a new function, organ ... organism, is a change (a breaking and a reconstruction) of the coherence structure, thus a change of the symmetries in the earlier organism. Like in physics, symmetry changes (thus breakings) and randomness seem to coexist also in life dynamics, but they affect the dynamics of the very phase space.

Our approach to the biological processes as extended critical transitions fits with this understanding of biological trajectories as cascades of symmetry changes Longo \& Montévil (2011a). Of course, this instability goes together with and is even an essential component of structural stability: each critical transitions is a symmetry change and it provides variability, diversity, thus adaptivity, at the core of biological viability. Even an individual organism is adaptive to a changing ecosystem, thus biologically robust, by the ever different re-generation of its parts. The sensitivity to minor fluctuations close to transition, which is a signature of critical phase transitions, enhances adaptivity of organisms (DNA methylation may affect even adaptive behavior, Kucharski et al. (2008)).

It seems thus impossible to extract relevant invariants concerning the specific structure of phenotypes and construct by them a space of all possible phenotypes. It may be even inadequate as variability is (one of) the main mathematical invariant in biology, beginning with individual mitoses. This does not forbid to propose some general invariants and symmetries, yet not referring to the specific aspects of the phenotype, as form and function. This is the path we followed when conceptualizing sufficiently stable properties, such as biological rhythms Bailly et al. (2011), extended criticality and anti-entropy (a tool for the analysis of biological complexity as anti-entropy, see Bailly \& Longo (2009; Longo \& Montévil (2012)).

We follow by this physics' historical experience of "objectivizing" by sufficiently stable concepts. In biology, these must encompas change and diversity. As a matter of fact, our investigations of biological rhythms, extended criticality and anti-entropy are grounded also on variability. In a long term perspective, these concepts should be turned all into more precisely quantified (and correlated) mathematical invariants and symmetries, in abstract spaces. This is what we did as for the two dimensional time of rhythms and as for anti-entropy, by imitating the way Schrödinger defined his equation in Hilbert spaces, far away from ordinary space-time. Abstract properties such as extended criticality and anti-entropy do not refer to the invariance of specific phenotypes, but they are themselves relatively stable, as they seem to refer to the few invariant properties of organisms. Their analysis, in a quantified space of extended criticality, may give us a better understanding of objects and trajectories within the ever changing space of phenotypes.

\section{Causes and Enablement}

We better specify now the notion of enablement, proposed in Longo et al. (2012b) and already used above. This notion may help to understand the role played by ecosystemic dynamics in the formation of a new observable (mathematically, a new dimension) of the phase space. Examples are given below and we will refine this notion throughout the rest of this paper.

In short, a niche enables the survival of an otherwise incompatible/impossible form of life, it does not cause it. More generally, niches enable what evolves, while evolving with it. At most, a cause may be found in the "difference" (a mutation, say) that induced the phenotypic variation at stake, as spelled out next.

This new perspective is motivated, on one side, by our understanding of physical "causes and determinations" in terms of symmetries, along the lines above of modern 
physics, and, on the other side, by our analysis of biological "trajectories" in phylogenesis (and ontogenesis), as continual symmetry changes. Note that, in spite of its modern replacement by the language of symmetries, the causal vocabulary still makes sense in physics: gravitation causes a body to fall (of course, Einstein's understanding in terms of geodetics in curved spaces, unifies gravitation and inertia, it is thus more general).

In biology, without sufficiently stable invariances and symmetries at the level of organisms, thus (possibly equational) laws, "causes" positively entailing the dynamics (evolution, typically) cannot be defined. As part of this understanding, we will discuss causal relations in a restricted sense, that is, in terms of "differential causes". In other words, since symmetries are unstable, causality in biology cannot be understood as "entailing causality" as in physics and this will lead us to the proposal that in biology, causal relations are only differential causes. If a bacterium causes pneumonia, or a mutation causes a monogenetic diseases (anemia falciformis, say), this is a cause and it is differential, i.e. it is a difference with respect to what is fairly considered "normal", "healthy" or "wild" as biologist say as for the genome, and it causes an anormality in the phenotype.

A classical mistake is to say: this mutation causes a mentally retarded child (a famous genetic disorder, phenylketonuria), thus ... the gene affected by the mutation is the gene of intelligence, or ... here is the gene that causes/determining the intelligence Weiss (1992) or encodes for (part of) the brain. In logical terms, this consists in deducing from "notA implies notB" that "A implies B" (or from "not normal A implies not normal B", that "normal A implies normal B"): an amazing logical mistake. All that we know is a causal correlation of differences ${ }^{12}$.

We then propose to consider things differently. The observed or induced difference, a mutation with a somatic effect, say, or a stone bumping on someone's head, or a carcinogen (asbestos), does cause a problem; that is, the causal dictionary is suitable to describe a differential cause - effect relation. The differential cause modifies the space of possibilities, that is the compatibility of the organism with the ecosystem. In other terms, it modifies the "enablement relations". This is for us, Longo et al. (2012b), the way an organism, a niche, an ecosystem may accommodate a phenotype, i.e. when the modified frame becomes viable for a new or different phenotype (a new organ or function, a differentiated organism).

We are forced to do so by the radical change of the default state in biology. Inertial movement, or, more generally, conservation principles in physics, need a force or an

\footnotetext{
${ }^{12}$ Schrödinger, in his 1944 book, was well aware of the limits of the differential analyses of the chromosomes and their consequences: "What we locate in the chromosome is the seat of this difference. (We call it, in technical language, a 'locus', or, if we think of the hypothetical material structure underlying it, a 'gene'.) Difference of property, to my view, is really the fundamental concept rather than property itself.", p.28.
}

efficient cause to change (see the revitalization of the Aristotelian distinction efficient/material cause, in Bailly \& Longo (2011)). In biology, default states guaranty change: reproduction with variation and motility, Longo et al. (2015). Causes "only" affect the intrinsic (the default) dynamics of organisms. More precisely, in our view, the differential causes modify the always reconstructed coherence structure of an organism, a niche, an ecosystem. So enablement is modified: a niche may be no longer suitable for an organism, an organism to the niche. Either selection may exclude the modified organism. Or a change in a niche, due to a differential physical cause (a climate change, for example), may negatively select existing organisms or enable the adaptive ones, since the enablement relations differ.

Differential analysis are crucial in the understanding of existing niches. Short descriptions of niches may be given from a specific perspective (they are strictly epistemic): they depend on the "purpose" one is looking at, say. And one usually finds out a feature in a niche by a difference, that is, by observing that, if a given feature goes away, the intended organism dies. In other terms, niches are compared by differences: one may not be able to prove that two niches are identical or equivalent (in enabling life), but one may show that two niches are different. Once more, there are no symmetries organizing over time these spaces and their internal relations.

In summary, while gradually spelling out our notion of esablement, we claim that only the differential relations may be soundly considered causal. Moreover, they acquire a biological meaning only in presence of enablement. In other words:

1. In physics, in presence of an explicit equational determination, causes may be seen as a formal symmetry breaking of equations. Typically, $f=m a$, a symmetric relation, means, for Newton, that a force, $f$, causes an acceleration $a$, asymmetrically. Thus, one may consider the application of a Newtonian force as a differential cause ${ }^{13}$. This is so, because the inertial movement is the "default" state in physics ("nothing happens" if no force is applied). This analysis cannot be globally transferred to biology, inasmuch symmetries are not stable and, thus, one cannot write equations for phylogenetic trajectories (nor break their symmetries). Moreover, the default state is far from being inertia (next point).

2. As just mentioned the default state in physics is inertia. In biology instead, the default state is "activity", as proliferation with variation and motility. As a consequence, an organism, a species, does not need a cause to be active, e.g. to reproduce with modi-

\footnotetext{
${ }^{13}$ In a synthetic/naive way, one may say that Einstein reversed the causal implication, as a space curvature "causes" an acceleration that "causes" a field, thus a force (yet, the situation is slightly more complicated and the language of symmetries and geodetics is the only rigorous one).
} 
ficatios and possibly occupy a new niche ${ }^{14}$. It only needs to be enabled in order to survive by changing. Moreover, in our terms, this default state involves continual critical transitions, thus symmetry changes, up to phase space changes.

Consider for example an adjacent possible empty niche, for example Kauffman's example of the swim bladder (see for example Kauffman (2002, 2012; Longo et al. (2012b)), formed by Gould's exaptation from the lung of some fishes. Is it a boundary condition? Not in the sense this term has in physics, since the swim bladder may enable a (mutated) worm or a bacterium to live and evolve, according to unpredictable enabling relations. That is, the observable features of the swim bladder to be used by the new organism to achieve functional closure in its environment may be radically new, possibly originating for both in a quantum based acausal/indeterminate molecular event and by correlated variations: the niche and the bacterium functionally shape each other. As discussed above, the combination of various forms of (physical and biological) randomness modify the set of observables (the new organ, the new bacterium), not just the values of some observables.

Once more, in physics, energy conservation properties allow us to derive the equations of the action/reaction system proper to the physical phenomenon in a pre-given phase space. Random event may modify the value of one of the pertinent observable, not the very set of observables. Typically, a river does co-constitute its borders by frictions, yet the observables and invariants to be preserved are well-know (energy and/or momentum), the game of forces as well. It may be difficult to write all the equations of the dynamics and some non-linear effects may give the unpredictability of the trajectory. Yet, we know that the river will go along a unique perfectly determined geodetics, however difficult it may be to calculate it exactly (to calculate the exact numerical values of the dynamics of the observables). Yet, a river never goes wrong and we know why: it will follow a geodetics. An onto- or phylogenetic trajectory may go wrong, actually most of the time it goes wrong. We are trying to theoretically understand "how it goes", between causes and enablement.

In summary, enablement and proliferation with variation and motility as default states are at the core of life dynamics. They conceptually frame the development of life in absence of a pre-definable phase space.

As we recalled, niches and phenotypes are co-constituted observables. Typically, the organism adjusting to / constructing a new niche may be a hopeful monster, that is the result of a "pathology" Dietrich (2003; Gould (1977). Now, notions of "normal" and "pathological" makes no sense in physics. They are contextual and historical in biology; they are contingent yet fundamental.

\footnotetext{
${ }^{14}$ Energy or matter, of course, is needed in order to reproduce, but it is not a cause. As we spell out in Bailly \& Longo (2011; Bailly et al. (2011), in biology energy is a parameter, like in allometric equations,
} it is not an "operator", like in physics.
These differing notions may also help to distinguish between enablement and causality, as the latter may be understood as a causal difference in the "normal" web of interactions. In evolution, a difference (a mutation) may cause a "pathology", as hopeful monster. That is, this monster, which is such with respect to the normal or wild phenotype, may be killed by selection or may be enabled to survive by and in a new co-constituted niche. A dark cavern may be modified, also as a niche for other forms of life, by the presence of the blind fish. And the contingent monster becomes the healthy origin of a speciation.

Thus, besides the centrality of enablement, we may maintain the notion of cause - and it would be a mistake to exclude it from the biological dictionary. As a matter of fact, one goes to the doctor and rightly asks for the cause of pneumonia - not only what enabled it: find and kill the bacterium, please, that is the cause. Yet, that bacterium has been enabled to grow excessively by a weak lung, a defective immune system or bad life habits ... Sंo, the therapy should not only concern the differential cause, the incoming bacteria, but investigate enablement as well. And good doctors do it, without necessarily naming it so, Noble (2009).

Finally, following Sonnenschein \& Soto (1999), by our approach we understand cancer as being enabled by a modified "society of cells" (the concerned tissue, organ, organism). A carcinogen affecting the organism (typically, the epithelial stroma, Sonnenschein \& Soto (1999)) deferentially modifies the "normal" tissue-niche for the cells and its coherence structure. The less controlled cells' default state, proliferation with variation, may then lead to the abnormal proliferation, possibly with increasing variation (as an elementary example, a teratoma has a larger number of cell types than a normal tissue).

\section{Structural stability, autonomy and constraints}

Organisms withstand the intrinsic unstability / unpredictability of the changing phase space, by the relative autonomy of their structural stability. They have an internal, permanently reconstructed autonomous coherent structure, Kantian wholes (in Kant's sense, see Kant (1781; Longo \& Perret (2013)), or Varela's autopoiesis, that gives them an ever changing, yet "inertial" structural stability — where inertia for organisms must be understood in the terms of biological protension, in Longo \& Montévil (2011b). They achieve a closure in a functional space by which they reproduce, and evolve and adapt by changing alone or together out of the indefinite and unorderable set of functions, or by finding new uses of preexisting components to sustain their activity in the ongoing co-evolution in the ecosystem.

The niche is indefinite in features prior to proliferation with variation and selection revealing what will coconstitute "task closure" for the organism. The niche allows the tasks' closure by which an organism survives and reproduces. 
Organisms and ecosystems are structurally stable, also because of their constrained autonomy, as they permanently and non-identically reconstruct themselves, their internal and external constraints. They do it in an always different, thus adaptive, way. They change the coherence structure, thus its symmetries. This reconstruction is thus random, but also not random, as it heavily depends on constraints, such as the proteins types imposed by the DNA, the relative geometric distribution of cells in embryogenesis, interactions in an organism, in a niche. Yet, the autopoietic activity is based also on the opposite of constraints: the relative autonomy of organisms. In other words, organisms transform the ecosystem while transforming themselves and they can stand this continual changes because they also have an internal preserved coherent structure (Bernard's "milieu interieur"). Its stability is maintained also by slightly, yet constantly changing internal symmetries, which enhance adaptivity, beginning with individual cellular mitosis in a mulitcellular organisms.

As we said, autonomy is integrated in and regulated by constraints, within an organism itself and of an organism within an ecosystem. Autonomy makes no sense without constraints and constraints apply to an autonomous unity. So constraints shape autonomy, which in turn modifies constraints, within the margin of viability, i.e. within the limits of the interval of extended criticality.

A way to understand the impossibility of a complete a priori description of actual and potential biological organisms and niches may be the following. Recall first the role of observable invariants and conservation properties in establishing physical phase spaces, since Galileo's inertia as a symmetry group. Then, recall how this allowed finite definitions, in terms of symmetries, of the most abstract infinite phase spaces. As a consequence of our analysis in terms of symmetry breakings, any given, possibly complete description of an ecosystem is incompressible, in the sense that any linguistic description may require new names and meanings for the new unprestatable functions. These functions and their names make only sense in the newly co-constructed biological and historical (even linguistic) environment. There is no way to define them a priori with finitely many words. The issue then is not infinity, but incompressibility by the lack of invariant symmetries, which we described in relation to extended criticality.

\section{Conclusion}

We stressed the role of invariance, symmetries and conservation properties in physical theories. Our preliminary aim has been to show that the powerful methods of physics that allowed to pre-define phase spaces on the grounds of the observables and the invariants in the "trajectories" (the symmetries in the equations) do not apply in biology.

An immense literature has been tackling "emergence" in life phenomena. Yet, in the technical analyses, the strong and dominating theoretical frames inherited from mathematical physics (or even computing) do not seem to have been abandoned. From Artificial Life, to Cellular Automata and various very rich analysis of dynamical systems, the frame for intelligibility is a priori given, under the form, often implicitly, of one or more pre-defined phase spaces, possibly to be summed up by adequate mathematical forms of products (Cartesian, tensorial ...). A very rich and motivated frame for these perspectives is summarized in Drake et al. (2007). Well beyond the many analysis which deal with equilibrium systems, an inadequate frame for biology, these authors deal with interactions between multiple attractors in dissipative dynamical systems, possibly given in two or more phase spaces (the notion of attractor is a beautiful mathematical notion, which requires explicit equations or evolution functions - solutions with no equations - in pertinent phase spaces in order to be soundly presented). Then, two deterministic, yet highly unpredictable and independent systems, which interact in the attractor space, may "produce persistent attractors that are offsprings of the parents .... Emergence in this case is absolute because no trajectories exist linking the child to either parent (p. 158) ... [The] source [of emergence] is the creation, evolution, destruction, and interaction of dynamical attractors (p. 179)".

This analysis is compatible with ours and it may enrich it by a further component, in pre-given interacting phase spaces. Yet, we go somewhat further by a critical perspective, which, per se, is a tool for intelligibility, and, below, we will hint again to further possible (and positive) work.

In our approach, the intrinsic unpredictability of the very Phase Space of biological processes is due, in summary, to:

1. extended criticality, as a locus for the correlation between symmetry breakings and randomness;

2. cascades of symmetry changes in (onto-) phylogenetic trajectories;

3. bio-resonance, due to interacting levels of organization, as a component both of integration and regulation, in an organism, as well as of amplification of random fluctuations in one level of organization through the others;

4. enablement, or the co-constitution of niches and phenotypes, a notion to be added to physical determination.

These phenomena are crucial also in order to understand life persistence, as they are at the origin of variability, thus of diversity and adaptability, which essentially contribute to biological structural stability. Our theoretical frame, in particular, is based on reproduction with modification and motility, as proper default states for the analysis of phylo- and ontogenenesis. This justifies the role of enablement, in particular.

More precisely, in biology, symmetries at the phenotypic level are continually changed, beginning with the least mitosis, up to the "structural bifurcations" which 
yield speciations in evolution. Thus, there are no biological symmetries that are a priori preserved, except and for some time, some basic structures such as bauplans (still more or less deeply modified during evolution). There are no sufficiently stable mathematical regularities and transformations, to allow an equational and law like description entailing the phylogenetic and ontogenetic trajectories. These are cascades of symmetry changes and thus just cumulative historical dynamics. And each symmetry change is associated to a random event (quantum, classical or due to bio-resonance), at least for the breaking of symmetries, while the global shaping of the trajectory, by selection say, is also due to non-random events. In this sense biological trajectories are generic, that is just possible ones, and yield a historical result, an individuated, specific organism (see Bailly \& Longo (2011; Longo \& Montévil (2011a)).

As a consequence, this sum of individuals and individualizing histories, co-constituted within an ever changing ecosystem, does not allow a compressed, finite or formal description of the space of possibilities, an actual biological phase space (functions, phenotypes, organisms): these possibilities are each the result of an unpredictable sequence of symmetry breakings, associated to random events, in contrast to the invariant (conservation) properties which characterize physical "trajectories", in the broad sense (extended to Hilbert's spaces, in Quantum Mechanics).

By the lack of mathematically stable invariants (stable symmetries), there are no laws that entail, as in physics, the observable becoming of the biosphere. The geodetic principle mathematically forces physical objects never to go wrong. A falling stone follows exactly the gravitational arrow. A river goes along the shortest path to the sea, it may adjust it by nonlinear well definable interactions as mentioned above, but it will never go wrong. These are all geodetics. Living entities, instead, go wrong most of the time: most organisms are extinct, almost half of fecundations, in mammals, do not lead to a birth, an amoeba does not follows, exactly, a curving gradient - by retention it would first go along the tangent, then correct the trajectory, in a protensive action. In short, life goes wrong most of the time, but it "adjusts" to the environment and changes the environment, if possible. It maintains itself, always in a critical transition, at each mitosis, that is within an extend critical interval, whose limits are the edge of death. It does so by changing the observables, the phenotypes and its niche, thus the very nature and space of the living object.

Then, we must ask new scientific questions and invent new tools, for this co-constitution by organisms as they co-evolve and make their worlds together. This must be seen as a central component of the biosphere's dynamics. The instability of theoretical symmetries in biology is not, of course, the end of science, but it sets the limits of the transfer of physico-mathematical methods, as taught us from Newton onward, to biology. In biological evolution we cannot use the same very rich interaction with mathematics as it has been constructed at the core of physical theories. However, mathematics is a human adaptive construction: an intense dialogue with biology may shape for it new scientific paths, concepts, structures, as it did with physics since Newton.

By providing some theoretical arguments that yield this "negative result", in terms of symmetries and critical transitions, we hope to have provided also some tools for a new opening. Negative results marked the beginning of new sciences in several occasions: the thermodynamic limit to energy transformation (increasing entropy), Poincaré's negative result (as he called his Three Body Theorem), Gödel's theorem (which set a new start to Recursion Theory and Proof Theory) all opened new ways of thinking, Longo (2012). Limits clarify the feasible and the non feasible with the existing tools and may show new directions by their very nature, if this has a sufficiently precise, scientific content.

The scientific answer we propose to this end of the physicalist certitudes, is based on our analysis of symmetry changes in extended critical transitions and on the notion of "enablement" in evolution (and ontogenesis). Enablement concerns how organisms co-create their worlds, with their changing symmetries and coherence structures, such that they can exist in a non-ergodic universe.

Following Longo et al. (2012b), our thesis then is that evolution as a "diachronic process" of becoming (but ontogenesis as well) "enables", but does not cause, unless differentially, the forthcoming state of affairs, in the sense specified above. Galileo and Newton's entailed trajectories mathematized Aristotle's "efficient cause" only. Instead, in our view, such entailed causal relations must be replaced by "enablement" relations, plus differential, often quantum indeterminate, causes, in biological processes.

Life is caught in a causal web, but lives also in a web of enablement and radical emergence of life from life, whose intelligibility may be largely given in terms of symmetry changes and their association to random events at all levels of organization.

We based our analysis on the Darwinian default state and key principles: reproduction with modification (plus motility) and selection. Selection shapes this bubbling forth of life by excluding the unfit. Our approach is just a further theoretical specification along these lines.

As hinted in 6, a long term project would be to better quantify our approaches to two dimensional time for rhythms, to extended criticality and to anti-entropy (see the references), in order to construct from them an abstract phase space based on these mathematically stable properties. The dynamical analysis should follow the nature of Darwin's evolution, which is an historical science, not meant at all to "predict", yet giving a remarkable knowledge of the living. Thus, the dynamics of extended criticality or anti-entropy should just provide the evolution of these state functions, or how these abstract observables may develop with respect to the intended parameters, in- 
cluding time. And this, without being "projectable" on specific phenotypes, even not in probabilities, as it is instead possible for Schrödinger's state functions in Quantum Mechanics. To this purpose, one should give a biologically interesting measure for extended criticality, as we did for anti-entropy Bailly \& Longo (2009), and describe in a quantitative way, in the abstract space of extended critical transitions, the qualitative evolution of live.

Now, going back to brain dynamics, the general framework we discussed has a number of consequences.

The first consequence concerns mathematical models following usual physical methodology, for example the model of the primary visual cortex as an implementation of a subriemannian geometry, corresponding to the visual field Petitot (2003). This model is biologically very relevant since it enables scientists to make sense of the functional architecture of the primary visual cortex. However, its geometry is only valid, stricto sensu, outside of an active brain, and outside of a living organism, as this cortex is closely connected and dependent with the rest of the organism in vivo Gandhi et al. (1999), and also because of the underlying variability in its intrinsic activity. In our more general vocabulary, the biological symmetries that allow to write this model are not stable and they are broken in many ways in vivo by the activity of the organism.

The second consequence, along the line of the work by Gerhard Werner, see for example Werner (2010, 2011), concerns the change of level of organization associated to critical-like phenomenon in the brain and more generally in the organism. This viewpoint emphasizes the relationship between scales, and the new level is not simply at a higher scale but across scales. In this frame, with respect to the question of consciousness and the classical Mindbody dichotomy, where "body" more or less means spatial extension, it is noteworthy that the renormalization viewpoint provides an original approach: phenomena are no longer understood as the combination of spatially extended interactions, as this combination may diverge (for small and/or large scales).

The third consequence concerns the changes of symmetry of the biological object. If we consider animal behaviours as a macroscopic aspect, the diversity in their pattern, both intra- and interspecific, is precisely an aspect of these symmetry changes that should not vanish from any analysis. This kind of considerations applies also to parts of the brain. Now, from a theoretical viewpoint, the key question is that of the objectivation of phenomena with such unstable symmetries. In particular, note that the usual physical renormalization relies mainly on an asymptotic symmetry of the equations by the change of scale, a symmetry that doesn't seem completely stable in biology. Note that a way to stabilize partially biological symmetries is to have an experimental setup that do so, this point will be discussed in a forthcoming article.

\section{Acknowledgments}

We would like to thank Stuart Kauffman for the very stimulating joint work that preceded this paper and the editors for their interest in "extended criticality". Longo's (and co-authors') papers are downloadable from http:// www.di.ens.fr/users/longo/.

Allin, E. (1975). Evolution of the mammalian middle ear. J. Morphol., 147, 403-437.

Arjun, R., \& van Oudenaarden, R. (2008). Stochastic gene expression and its consequences. Cell, 135, 216-226.

Aspect, A., Grangier, P., \& Roger, G. (1982). Experimental realization of Einstein-Podolsky-Rosen-Bohm Gedankenexperiment: A new violation of Bell's inequalities. Physical Review Letters, 49, 91-94. doi:10.1103/PhysRevLett.49.91.

Bailly, F. (1991). L'anneau des disciplines. Revue Internationale de Systémique, 5.

Bailly, F., Gaill, F., \& Mosseri, R. (1988). Fonctions biologiques, niveaux d'organisation et dimensions fractales. Revue Internationale de Systémique, 2.

Bailly, F., \& Longo, G. (2008). Extended critical situations: the physical singularity of life phenomena. Journal of Biological Systems, 16, 309. doi:10.1142/S0218339008002514.

Bailly, F., \& Longo, G. (2009). Biological organization and antientropy. Journal of Biological Systems, 17, 63-96. doi:10.1142/ S0218339009002715.

Bailly, F., \& Longo, G. (2011). Mathematics and the natural sciences; The Physical Singularity of Life. London: Imperial College Press. Preliminary version in French: Hermann, Vision des sciences, 2006

Bailly, F., Longo, G., \& Montévil, M. (2011). A 2-dimensional geometry for biological time. Progress in Biophysics and Molecular Biology, 106, 474-484. doi:10.1016/j . pbiomolbio.2011.02.001.

Batterman, R. W. (2007). Intertheory relations in physics. URL: http://plato. stanford.edu/entries/physics-interrelate/ first published in 2001

Brouzés, E., \& Farge, E. (2004). Interplay of mechanical deformation and patterned gene expression in developing embryos. Current Opinion in Genetics 86 Development, 14, 367 - 374. doi:10.1016/ j.gde. 2004.06.005.

Buiatti, M., \& Longo, G. (2013). Randomness and multilevel interactions in biology. Theory in Biosciences, 132, 139-158. URL: http://dx.doi.org/10.1007/s12064-013-0179-2. doi:10 . 1007/s12064-013-0179-2.

Ceron-Carrasco, J., Requena, A., Perpete, E., \& Michaux, D., C.and Jacquemin (2009). Double proton transfer mechanism in the adenine-uracil base pair and spontaneous mutation in rna duplex. Chemical Physics Letters, 484, 64-68.

Collini, E., Wong, C., Wilk., K., Curmi, P., Brurner, P., \& Scholes G., D. (2010). Coherently wired light harvesting in photosynthetic marine algae at ambient temperature. Nature, 463, 644-648.

Dietrich, M. (2003). Richard goldschmidt: hopeful monsters and other 'heresies'. Nature reviews. Genetics, 4, 68-74. Historical Article,Journal Article,Portraits,

Drake, J., Fuller, M., Zimmermann, C., \& Gamarra, J. (2007). From energetics to ecosystems: the dynamics and structure of ecological systems. Springer.

Einstein, A., Podolsky, B., \& Rosen, N. (1935). Can quantummechanical description of physical reality be considered complete? Physical Review, 47, 777-780. doi:10.1103/PhysRev. 47.777.

Engel, G., Calhoun, T., Read, E., Ahn, T., Mançal, T., Yuan-Chung, C., Blankenship, R., \& Fleming, G. (2007). Evidence for wavelike energy transfer through quantum coherence in ptotosynthetic systems. Nature, 447, 782-786.

Fleury, V. (2000). Branching morphogenesis in a reaction-diffusion model. Phys. Rev. E, 61, 4156 - 4160. doi:10.1103/PhysRevE. 61. 4156.

Gandhi, S. P., Heeger, D. J., \& Boynton, G. M. (1999). Spatial 
attention affects brain activity in human primary visual cortex Proceedings of the National Academy of Sciences, 96, 3314-3319.

Gould, S. (1977). The return of hopeful monsters. Natural History, $86,22-30$.

Gray, H., \& Winkler, J. (2003). Electron tunneling through proteins. Q. Rev. Biophys., 36, 341-372.

Heams, T. (2014). Randomness in biology. Math. Structures in Comp. Sci., special issue, 24.

Jean, R. (1994). Phyllotaxis: A Systemic Study in Plant Morphogenesis. Cambridge Studies in Mathematics.

Jensen, H. J., \& Sibani, P. (2007). Glassy dynamics. Scholarpedia, 2, 2030.

Kant, I. (1781). Critique of Pure Reason. transl. N. Kemp Smith, Palgrave Macmillan, this edition published 1929.

Kauffman, S. (2002). Investigations. Oxford University Press, USA.

Kauffman, S. (2012). The end of a physics worldview. URL: http://www.mylab.fi/en/statement/the_end_of_a_physics_ worldview/.

Kucharski, R., Maleszka, R., Foret, S., \& Maleszka, A. (2008). Nutritional control of reproductive status in honeybees via dna methylation. Science, 319, 1827-1830.

Kupiec, J. (1983). A probabilistic theory of cell differentiation, embryonic mortality and dna c-value paradox. Specul. Sci. Techno. $6,471-478$.

Laskar J., . (1994). Large scale chaos in the solar system. Astron. Astrophys., 287.

Lesne, A. (2008). Robustness: Confronting lessons from physics and biology. Biol Rev Camb Philos Soc, 83, 509 - 532. doi:10.1111/ j.1469-185X.2008.00052.x.

Liu, R., \& Ochman, H. (2007). Stepwise formation of the bacterial flagellar system. Proceedings of the National Academy of Sciences, 104, 7116 - 7121. doi:10.1073/pnas. 0700266104 .

Longo, G. (2011). Mathematical infinity "in prospettiva" and the spaces of possibilities. in "visible", a Semiotics Journal, 9.

Longo, G. (2012). On the relevance of negative results. In Conference on Negation, duality, polarity, Marseille 2008. URL: http://www. influxus.eu/article474.html (proceedings inInfluxus, electronic journal ).

Longo, G., Miquel, P.-A., Sonnenschein, C., \& Soto, A. M. (2012a). Is information a proper observable for biological organization? Progress in Biophysics and Molecular biology, 109, 108 - 114. doi:10.1016/j.pbiomolbio.2012.06.004.

Longo, G., \& Montévil, M. (2011a). From physics to biology by extending criticality and symmetry breakings. Progress in Biophysics and Molecular Biology, 106, 340 - 347. doi:10.1016/j. pbiomolbio.2011.03.005. Invited paper, special issue: Systems Biology and Cancer.

Longo, G., \& Montévil, M. (2011b). Protention and retention in biological systems. Theory in Biosciences, 130, 107-117. doi:10. 1007/s12064-010-0116-6.

Longo, G., \& Montévil, M. (2012). Randomness increases order in biological evolution. In LNCS, Proceedings of the colloquium in honor of Christian Calude, Auckland. Springer-Verlag. Submitted.

Longo, G., \& Montévil, M. (2013). Extended criticality, phase spaces and enablement in biology. Chaos, Solitons \& Fractals, 55, 64 - 79. doi:10.1016/j.chaos.2013.03.008. Invited Paper, Special Issue.

Longo, G., Montévil, M., \& Kauffman, S. (2012b). No entailing laws, but enablement in the evolution of the biosphere. In Genetic and Evolutionary Computation Conference. GECCO'12 New York, NY, USA: ACM. doi:10.1145/2330784.2330946 invited Paper.

Longo, G., Montévil, M., \& Pocheville, A. (2012c). From bottom-up approaches to levels of organization and extended critical transitions. Frontiers in Physiology, 3. doi:10.3389/fphys.2012.00232. Invited paper.

Longo, G., Montévil, M., Sonnenschein, C., \& Soto, A. M. (2015). In search of principles for a theory of organisms. Journal of Biosciences, (pp. 1-14). doi:10.1007/s12038-015-9574-9.

Longo, G., Palamidessi, C., \& Paul, T. (2010). Some bridging results and challenges in classical, quantum and computational random- ness. In H. Zenil (Ed.), Randomness through Computation. World Scientific

Longo, G., \& Perret, N. (2013). Anticipation, protention and biological inertia. to appear,

Lovecchio, E., Allegrini, P., Geneston, E., West, B. J., \& Grigolini, P. (2012). From self-organized to extended criticality. Frontiers in Physiology, 3. URL: http://www.frontiersin.org/fractal_ physiology/10.3389/fphys.2012.00098/abstract. doi:10.3389/ fphys. 2012.00098.

Machta, B. B., Papanikolaou, S., Sethna, J. P., \& Veatch, S. L. (2011). A minimal model of plasma membrane heterogeneity requires coupling cortical actin to criticality. Biophysical journal, 100, 1668-1677. doi:10.1016/j.bpj.2011.02.029.

Michl, M., Ouyang, N., Fraek, M.-L., Beck, F.-X., \& Neuhofer, W. (2006). Expression and regulation of $\alpha \beta$-crystallin in the kidney in vivo and in vitro. Pflügers Archiv, 452, 387-395. doi:10.1007/ s00424-005-0033-6.

Mossio, M., \& Moreno, A. (2010). Organisational closure in biological organisms. History and philosophy of the life sciences, 32, 269288

Noble, D. (2009). Could there be a synthesis between western and oriental medicine, and with sasang constitutional medicine in particular? Evidence-Based Complementary and Alternative Medicine, 6, 5-10. doi:10.1093/ecam/nep101.

Paldi, A. (2003). Stochastic gene expression during cell differentiation: order from disorder? Cell Mol. Life Sci., 60, 1775-1779.

Petitot, J. (2003). The neurogeometry of pinwheels as a subriemannian contact structure. Journal of Physiology, Paris, 97 , 265-309. doi:10.1016/j.jphysparis.2003.10.010.

Robert, L., Paul, G., Chen, Y., Taddei, F., Baigl, D., \& Lindner, A. B. (2010). Pre-dispositions and epigenetic inheritance in the escherichia coli lactose operon bistable switch. Mol Syst Biol, 6 . doi:10.1038/msb. 2010.12.

Sethna, J. P. (2006). Statistical mechanics: Entropy, order parameters, and complexity. New York: Oxford University Press.

Sonnenschein, C., \& Soto, A. (1999). The society of cells: cancer and control of cell proliferation. New York: Springer Verlag.

Strocchi, F. (2005). Symmetry breaking volume 732 of Lecture Notes in Physics. Heidelberg: Springer Verlag. doi:10.1007/b95211.

Van Fraassen, B. (1989). Laws and symmetry. Oxford University Press, USA.

Varela, F., Maturana, H., \& Uribe, R. (1974). Autopoiesis: The organization of living systems, its characterization and a model. Biosystems , 5, 187 - 196. doi:10.1016/0303-2647(74)90031-8.

Weiss, V. (1992). Major genes of general intelligence. Personality and Individual Differences, 13, 1115 - 1134. doi:10.1016/ 0191-8869 (92) 90026-L.

Werner, G. (2010). Fractals in the nervous system: conceptual implications for theoretical neuroscience. Frontiers in Physiology, 1. doi:10.3389/fphys. 2010.00015.

Werner, G. (2011). Consciousness viewed in the framework of brain phase space dynamics, criticality, and the renormalization group. arXiv:1103.2366, . URL: http://arxiv.org/abs/1103.2366.

West, B. (2006). Where medicine went wrong: Rediscovering the path to complexity volume Volume 11 of Studies of nonlinear phenomena in life sciences. Teaneck, NJ: World Scientific.

Weyl, H. (1983). Symmetry. Princeton: Princeton University Press.

Winkler, J. R., Gray, H. B., Prytkova, T. R., Kurnikov, I. V., \& Beratan, D. N. (2005). Electron transfer through proteins. In Bioelectronics (pp. 15-33). Wiley-VCH Verlag GmbH and Co. KGaA. doi:10.1002/352760376X.ch2. 